\title{
Propuesta de Competencias Emocionales y solución de conflictos en estudiantes de una universidad
}

Proposal for Emotional Competencies and conflict resolution in university students Proposta de Competências Emocionais e Resolução de Conflitos em Estudantes Universitários.

\section{ARTÍCULO GENERAL}

Dennís Yvonne Del Pino Ascona

c20345@utp.edu.pe

https://orcid.org/0000-0001-7360-1251

Universidad Tecnológica del Perú, Ica - Perú
Hugo Nuñez Roman

h_nunez_roma@doc.uap.edu.pe

https://orcid.org/0000-0002-4456-8621

Universidad Alas Peruanas, Ica - Perú

Recibido 16 de Diciembre 2020 | Arbitrado y aceptado 06 de Enero 2021 | Publicado el 01 de Marzo 2021

\section{RESUMEN}

Esta investigación genera un espacio para aportar al esclarecimiento y explicación de algunos problemas educativos como es; los conflictos intrapersonales del estudiante, lo que permite implementar acciones de mejora de la calidad educativa del país. Para llevar a cabo esta investigación se tuvo en cuenta los siguientes antecedentes:

Tobalino (2002), con su investigación titulada: Relación que existe entre la competencia emocional y el rendimiento académico en los estudiantes de la Facultad de Educación de la Universidad Nacional Enrique Guzmán y Valle. Este trabajo demostró que el nivel de competencia emocional de los estudiantes de la especialidad de educación inicial de la Universidad de educación "Enrique Guzmán y Valle" se correlaciona con su rendimiento académico.

\section{ABSTRACT}

This research generates a space to contribute to the clarification and explanation of some educational problems such as; the intrapersonal conflicts of the student, which allows implementing actions to improve the educational quality of the country. In carrying out this research, the following information was taken into account:

Tobalino (2002), with his research titled: Relationship between emotional competence and academic performance in students of the Faculty of Education of the Enrique Guzmán y Valle National University. This work demonstrated that the level of emotional competence of the students of the specialty of initial education of the University of Education "Enrique Guzmán y Valle" is correlated with their academic performance.

\section{RESUMO}

Esta pesquisa gera um espaço para contribuir com o esclarecimento e explicação de alguns problemas educacionais como; os conflitos intrapessoais do aluno, o que permite implementar ações para melhorar a qualidade educacional do país. Para a realização desta pesquisa, as seguintes informações foram levadas em consideração:

Tobalino (2002), com sua pesquisa intitulada: Relação entre competência emocional e desempenho acadêmico em alunos da Faculdade de Educação da Universidade Nacional Enrique Guzmán y Valle. Este trabalho demonstrou que o nível de competência emocional dos alunos da especialidade de educação inicial da Universidade de Educação "Enrique Guzmán y Valle" está correlacionado com seu desempenho acadêmico. 


\section{Introducción}

\section{Objetivos e hipótesis}

Se tubo por objetivo: Determinar en qué medida la propuesta de competencias emocionales influye en la solución de conflictos en estudiantes de la Escuela Profesional de Ingeniería Ambiental de la Universidad Alas Peruanas, Sede Pisco, en el año 2015.

La hipótesis planteada fue: La propuesta de competencias emocionales influye significativamente en la solución de conflictos en estudiantes de la Escuela de Ingeniería Ambiental de la Universidad Alas Peruanas de Pisco en el año 2015.

\section{Método}

\section{Participantes}

La población estuvo constituida por 115 estudiantes de la Escuela de Ingeniería Ambiental de la Universidad Alas Peruanas de Pisco; la muestra quedó conformada por 45 estudiantes de los cuales 20 estudiantes del IV ciclo pertenecen al grupo de control y 25 estudiantes del VI ciclo pertenecen al grupo experimental. La elección de las unidades de la muestra fue mediante el muestreo no probabilístico. Los integrantes de la muestra de estudio fueron codificados para garantizar el tratamiento anónimo de la información y preservar la integridad de las unidades muéstrales.

La información presentada en el marco teórico se ha estructurado respetando el derecho a la propiedad intelectual para tal efecto se han utilizado las citas textuales considerando las especificaciones técnicas.

\section{.Instrumentos}

Para la recolección de los datos se diseñó como instrumento un cuestionario sobre el manejo de solución de conflictos estructurado en 24 ítems, que responden a las dimensiones: Prevención; análisis y negociación; mediación. Con criterio de valoración de los ítems de siempre ( 2 puntos), a veces (1 punto) y nunca (0 puntos). Los resultados de la variable Solución de conflictos se categorizaron en Inadecuado [0-16>, medianamente adecuado [16-32>, Adecuado [32-48]. 
Para determinar la prueba de Fiabilidad interna del Instrumento se utilizó el coeficiente de Alfa de Cronbach después de aplicado el Instrumento a la muestra piloto.

\section{Procedimiento}

A los estudiantes seleccionados se les aplicó un cuestionario sobre el manejo de solución de conflicto antes y después de la aplicación del estímulo académico (Sesiones de aprendizaje), con la finalidad de determinar el nivel de mejora alcanzado por los estudiantes en el manejo de solución de conflictos, mediante la comparación de resultados.

Para aplicar la Propuesta de competencias emocionales (estímulo académico), se utilizaron 11 sesiones de aprendizaje de aproximadamente 2 horas cada una. La metodología de su contenido se basó según indica (Segovia, 2010), en la participación y la comprensión. Se busca que los estudiantes participen en la realización de las actividades, y comprendan que es lo que se realiza en todo momento mediante una participación activa, estimando el protagonismo de los estudiantes para una mayor motivación e implicación en la realización de los ejercicios. Los estudiantes son los creadores del conocimiento a través de las reflexiones llevadas, fomentándose las relaciones sociales y la educación en valores, sobre todo favoreciendo las conductas positivas y rechazando las conductas negativas como la falta de respeto, insultos etc.

Las actividades desarrolladas fueron agrupadas por bloques, y se presentan el la siguiente tabla.

Tabla 1. Metodología del contenido de la Propuesta de Competencias Emocionales

\begin{tabular}{|c|c|c|c|c|}
\hline Bloque & Título de bloque & Actividad & $\begin{array}{l}\text { Título de } \\
\text { actividad }\end{array}$ & Objetivos didácticos \\
\hline \multirow{4}{*}{ I } & \multirow{4}{*}{$\begin{array}{l}\text { Conciencia } \\
\text { Emocional }\end{array}$} & & El & Identificar, clasificar y describir diferentes \\
\hline & & 01 & Vocabulario & emociones; y utilizar y ampliar el \\
\hline & & & Emocional & vocabulario emocional. \\
\hline & & 02 & $\begin{array}{l}\text { La caja de } \\
\text { emociones }\end{array}$ & $\begin{array}{l}\text { Utilizar le vocabulario emocional; adquirir } \\
\text { conciencia de las distintas emociones; } \\
\text { distinguir emociones. }\end{array}$ \\
\hline
\end{tabular}


Relajando el cuerpo

El semáforo

de las emociones

Experimentar la relajación; describir el bienestar que ofrece la relajación; valorar la relajación como un estado que nos permite regular las emociones; adquirir la práctica de la relajación.

Identificar emociones; reconocer y aceptar las emociones experimentadas; analizar y valorar la respuesta más apropiada; regular las emociones.

Identificar diferentes niveles de intensidad de las emociones; reflexionar sobre posibles consecuencias que desembocan

No te los niveles de intensidad; evitar el descontroles. descontrol; analizar situaciones en que se han manifestado diferentes niveles de intensidad emocional; aceptar y experimentar emociones.

Estimular el desarrollo de la autoestima a través de conocimiento personal; identificar características personales;

Así soy yo. estimular la valoración de la propia individualidad mediante la diferenciación personal; expresar características propias verbalmente.

Aceptar, reconocer y experimentar Mi propia cueva. pensamientos y emociones propias; expresar pensamientos y emociones; comparar estados interiores, previos y posteriores a la actividad.

Reconocer maneras de saludar y despedirse; identificar el saludo y la

Habilidades socioemocionales

Habilidades de vida Encuentros despedida en función de las personas; predisponer al otro para la relación; identificar emociones en las diferentes actitudes de saludo y despedida.

Los demás también sienten

Cometa de amistad.
Favorecer la aceptación de los demás; detectar las emociones ajenas; buscar alternativas.

Identificar las características que valoramos de nuestros amigos; interesarse por ser un buen amigo; construir el concepto de amistad; valorar el concepto de amistad. 
Yo hablo, tú Apreciar la necesidad de escuchar; no me descubrir el sentimiento de sentirse escuchas. ignorado.

\section{Análisis de datos}

Se inició al concluir la recolección de los datos. En posesión de un cúmulo de información, lo siguiente fue su organización y análisis considerando los siguientes pasos: Codificación. La información fue comparada y se generó códigos para cada uno de los sujetos muéstrales de acuerdo a las pruebas pre test y post test.

Calificación. Consistió en la asignación de un puntaje o valor según los criterios establecidos en la matriz del instrumento para la recolección de datos. En el caso de las respuestas de los ítems del cuestionario para el manejo de solución de conflictos, se le asignó los siguientes valores: siempre ( 2 puntos), a veces ( 1 punto) y nunca ( 0 puntos).

En el proceso de la tabulación de datos se elaboró una data donde se encuentren todos los códigos de los sujetos muéstrales y en su calificación se aplicó estadígrafos como las frecuencias, que permitieron conocer cuáles son las características de la distribución de los datos, por la naturaleza de la investigación se utilizó la media aritmética y desviación estándar.

En la etapa de la interpretación de los datos, estos se presentaron en tablas y figuras que fueron interpretados en función de la variable.

La comprobación de la hipótesis se trabajó a través de los métodos estadísticos. Se tuvo en cuenta la prueba de $\mathrm{T}$ de student por ser una muestra paramétrica y menor a 30 datos.

\section{Resultados}

El Coeficiente de Alfa de Cronbach obtenido para determinar la confiabilidad del instrumento dio como resultado que: $\alpha=0,921$; este dato señala que el instrumento para 
evaluar tiene un alto grado de confiabilidad situación que indica que el instrumento realiza mediciones estables en torno a la variable solución de conflictos.

El resultado del análisis de datos se presenta en las siguientes tablas:

Tabla 2. Solución de conflictos en estudiantes de la Escuela de Ingeniería Ambiental de la Universidad Alas Peruanas de Pisco en el año 2015, prueba pretest.

\begin{tabular}{lllll}
\hline & \multicolumn{2}{l}{ Grupo experimental } & \multicolumn{2}{l}{ Grupo de control } \\
\hline Categorías & $\mathrm{f}(\mathrm{i})$ & $\mathrm{h}(\mathrm{i}) \%$ & $\mathrm{f}(\mathrm{i})$ & $\mathrm{h}(\mathrm{i}) \%$ \\
Deficiente [0-16> & 5 & $20 \%$ & 6 & $30 \%$ \\
Regular [16-32> & 19 & $76 \%$ & 13 & $65 \%$ \\
Bueno [32-48] & 1 & $4 \%$ & 1 & $5 \%$ \\
Total & 25 & $100 \%$ & 20 & $100 \%$ \\
\hline
\end{tabular}

Fuente: Data de resultados obtenidos de la aplicación de la cuestionario sobre solución de conflictos

En la tabla 2, se observa en el grupo experimental a 19 estudiantes que representan el $76 \%$ de la muestra de estudio presentan un nivel regular de solución de conflictos y 1 estudiante que representa el $4 \%$ de la muestra de estudio presenta un buen nivel de solución de conflictos.

Por otro lado se observa en la tabla los resultados con respecto al grupo de control: 6 estudiantes que representan el 30\% de la muestra de estudio presentan un deficiente nivel de solución de conflictos, 13 estudiantes que representan el 65\% de la muestra de estudio presentan un nivel regular de solución de conflictos y 1 estudiante que representa el 5\% de la muestra de estudio presenta un buen nivel de solución de conflictos.

Se puede concluir señalando que ambos grupos en cuanto a su nivel de solución de conflictos se encuentran en igual condición antes de la aplicación de la propuesta de competencias emocionales en las sesiones de aprendizaje.

En conclusión; solo el 5\% de estos estudiantes tienen un nivel bueno de manejo de conflictos. 
Tabla 3. Solución de conflictos en estudiantes de la Escuela de Ingeniería Ambiental de la Universidad Alas Peruanas de Pisco en el año 2015, prueba postest.

\begin{tabular}{|c|c|c|c|c|}
\hline & \multicolumn{2}{|c|}{ Grupo experimental } & \multicolumn{2}{|c|}{ Grupo de control } \\
\hline Categorías & f(i) & $h(i) \%$ & $f(i)$ & $h(i) \%$ \\
\hline Deficiente $[0-16>$ & 0 & $0 \%$ & 2 & $10 \%$ \\
\hline Regular [16-32> & 3 & $12 \%$ & 17 & $85 \%$ \\
\hline Bueno [32-48] & 22 & $88 \%$ & 1 & $5 \%$ \\
\hline Total & 25 & $100 \%$ & 20 & $100 \%$ \\
\hline
\end{tabular}

Fuente: Data de resultados obtenidos de la aplicación de la cuestionario sobre solución de conflictos

En la tabla 3, se observa los resultados de la evaluación post test. Con respecto al grupo experimental, ningún estudiante presenta un deficiente nivel de solución de conflictos, 3 estudiantes que representan el 12\% de la muestra de estudio presentan un nivel regular de solución de conflictos y 22 estudiantes que representa el $88 \%$ de la muestra de estudio presentan un buen nivel de solución de conflictos.

Por otro lado se observa en la tabla los resultados con respecto al grupo de control; 2 estudiantes que representan el $10 \%$ de la muestra de estudio presentan un deficiente nivel de solución de conflictos, 17 estudiantes que representan el 85\% de la muestra de estudio presentan un nivel regular de solución de conflictos y 1 estudiante que representa el $5 \%$ de la muestra de estudio presenta un buen nivel de solución de conflictos.

Se puede concluir señalando que el grupo experimental en esta postprueba ha demostrado mejor manejo de los conflictos en comparación con el otro grupo que no recibió el estímulo académico.

De manera general se puede señalar que los estudiantes del grupo de control presentan un incremento poco significativo en las dimensiones evaluadas debido a que no participaron en la aplicación de la propuesta de competencias emocionales en las sesiones de aprendizaje.

\section{Discusión y Conclusiones}


En esta investigación al determinar en qué medida la propuesta de competencias emocionales influye en la solución de conflictos en estudiantes de la Escuela de Ingeniería Ambiental de la Universidad Alas Peruanas de Pisco en el año 2015 se encontró un T calculado de tc $=10,94$ pertenece a la región de rechazo. Esto quiere decir que la propuesta de competencias emocionales influyó significativamente en la solución de conflictos en los estudiantes que recibieron el estímulo académico (sesión de aprendizajes). Frente a lo mencionado se rechazó la Ho y en consecuencia se aceptó la Ha, se puede afirmar con un nivel de confianza del 95\% y significancia del 5\% que la propuesta de competencias emocionales influye significativamente en la solución de conflictos en estudiantes de la Escuela de Ingeniería Ambiental de la Universidad Alas Peruanas de Pisco en el año 2015.

Los resultados se contrastan con otras investigaciones llevadas a cabo entre ellos se tiene a Chacaltana y Cucho (2013), con su investigación titulada: Aplicación de estrategias de solución de conflictos para mejorar las relaciones interpersonales, en donde concluyeron que Los datos consignados muestran que los alumnos del grupo experimental logran un incremento aceptable de $36.75 \%$ en su nivel de control emocional. Por otro lado se tiene la información presentada en el marco teórico respecto a la dimensión prevención de la solución de conflictos según Cascón (2000), lo define como el proceso de intervención y regulación del conflicto cuando está en sus primeros estadios, antes de que aumente la tensión.

De lo expuesto se puede concluir que el grupo experimental ha mejorado su nivel de comprensión y negociación de la solución de conflictos debido a la aplicación de la propuesta de competencias emocionales; mientras que el grupo de control no obtuvo la mejora.

\section{Referencias}

Alzate, R. (1998). Análisis y resolución de conflictos. Una perspectiva psicológica. Bilbao: Universidad del País Vasco.

Beltrán, J. (2002) Las claves psicológicas de la convivencia escolar. Ponencia. Seminario Convivencia en los centros escolares como factor de calidad: Madrid. 
Bisquerra, R. (2000). Educación emocional y bienestar. Barcelona: Ciss- Praxis.

Bisquerra, R. (2011). Educación emocional. Propuestas para educadores y familias. Bilbao: Desclee de Brouwer.

Bisquerre, R. (2003). Educación emocional y competencias básicas para la vida. Revista de investigación Educativa (RIE), 21, 1, 7-43

Calhoun, C., y Solomon. R. (1989). ¿Qué es una emoción? Mexico: Fondo de Cultura Económica.

Caperna, A. (2010) Desarrollo de las competencias emocionales en el marco escolar. CEE Participación Educativa, 15, noviembre 2010 (pp. 40-57).

Cascón, P. (2000) La mediación. Cuadernos de pedagogía. Núm. 287 (pp. 72 -76).

Chacaltana, J. y Cucho, N. (2013). Aplicación de estrategias de solución de conflictos para mejorar las relaciones interpersonales de los estudiantes del quinto grado de educación secundaria de la Institución Educativa "José Carlos Mariátegui" de San Clemente-Pisco, 2011. Tesis de maestría de la Universidad César Vallejo sede Ica.

De Souza, L. (2012). Competencias emocionales y resolución de conflictos interpersonales en el aula, Universidad Autónoma de Barcelona - España. 